\title{
Características físico-químicas de sucos integrais elaborados a partir de uvas da espécie Vitis rotundifolia
}

\section{Physicochemical characteristics of whole grape juices elaborated from Vitis rotundifolia species}

Angélica Bender ${ }^{1 *}$ (D), André Luiz Kulkamp de Souza², Vinicius Caliari², Edson Luiz de Souza ${ }^{3}$, Marcelo Barbosa Malgarim¹, Samila Silva Camargo ${ }^{4}$

${ }^{1}$ Universidade Federal de Pelotas (UFPel), Capão do Leão/RS - Brasil

${ }^{2}$ Empresa de Pesquisa e Extensão Rural de Santa Catarina (Epagri), Videira/SC - Brasil

${ }^{3}$ Universidade do Oeste de Santa Catarina (UNOESC), Videira/SC - Brasil

${ }^{4}$ Serviço Nacional de Aprendizagem Industrial (SENAI), Videira/SC - Brasil

*Corresponding Author: Angélica Bender, Universidade Federal de Pelotas (UFPel), Campus Universitário, s/n, CEP: 96160-000, Capão do Leão/RS - Brasil, e-mail: bender.angelica.fruti@gmail.com

Cite as: Bender, A., Souza, A. L. K., Caliari, V., Souza, E. L., Malgarim, M. B., \& Camargo, S. S. (2019).

Physicochemical characteristics of whole grape juices elaborated from Vitis rotundifolia species. Brazilian Journal of

Food Technology, 22, e2018310. https://doi.org/10.1590/1981-6723.31018

\begin{abstract}
Resumo
Objetivou-se com este trabalho avaliar o perfil físico-químico de sucos elaborados com uvas das variedades Vitis rotundifolia Regale, Noble, Summit e Carlos. O trabalho foi realizado na Epagri-Videira/SC, nas safras 2016, 2017 e 2018. O delineamento experimental utilizado foi um fatorial duplo (variedade $\times$ safra), de forma inteiramente casualizada e com três repetições. As análises físico-químicas realizadas foram: acidez total (AT), teor de sólidos solúveis (SS), relação SS/AT, $\mathrm{pH}$, açúcares redutores, densidade e cor. Em relação ao pH, 'Regale' e 'Carlos' apresentaram valores que se mantiveram na faixa de 2,82 a 2,98, enquanto para 'Noble' e 'Summit', os valores foram superiores, chegando a 3,30. A acidez titulável dos sucos de 'Regale' e 'Noble' se destacou das demais variedades em ambas as safras avaliadas, devido aos teores de acidez mais elevados. Para o teor de sólidos solúveis totais, na safra 2016, apenas os sucos da variedade 'Summit' atingiram o valor de 14,00 'Brix, teor mínimo exigido para comercialização como suco integral perante a legislação brasileira. Os sucos dessa variedade obtiveram a maior relação SS/AT nas três safras avaliadas, com valores próximos a 30, comportamento diverso dos sucos de 'Regale', que apresentaram as menores médias para esta variável, com valores em torno de 10 . As variedades tintas originaram sucos mais ácidos em comparação às variedades brancas. A variedade 'Summit' apresentou a melhor relação açúcar/acidez, apresentando aptidão para a elaboração de sucos nas três safras pesquisadas. 'Regale' e 'Noble' apresentaram prejuízos na produtividade e sanidade na safra 2016, no entanto, mostraram-se aptas ao processamento de suco nas safras 2017 e 2018. Os sucos da variedade 'Carlos' não atingiram o teor mínimo de 14 Brix em nenhuma das três safras estudadas, fator que impossibilita a classificação destes como suco integral.
\end{abstract}

Palavras-chave: Muscadínea; Processamento; Composição; Qualidade; Safras; Elaboração. 


\begin{abstract}
The Vitis rotundifolia grape species have a very characteristic aroma and flavour and are used in the elaboration of juices, jellies and fermented products, as well as their in natura consumption in the United States. In Brazil, however, they are still little known. Due to the potential of the muscadine grapes for the diversification of viticulture and the scarcity of data concerning the quality of their juice, the objective of this work was to evaluate the physical and chemical profile of juices elaborated with the Vitis rotundifolia grape varieties: Regale, Noble, Summit and Carlos. The study was carried out in Epagri-Videira/SC, Brazil with the 2016, 2017 and 2018 harvests. A double factorial (variety $\mathrm{x}$ harvest) experimental design was used in a completely randomized format with three replications. The physicochemical analyses carried out were: total acidity (TA), soluble solids content (SS), SS/TA ratio, $\mathrm{pH}$, reducing sugars, density and colour. In relation to $\mathrm{pH}$, 'Regale' and 'Carlos' presented values that remained in the range from 2.82 to 2.98 , while the values were higher for 'Noble' and 'Summit', reaching 3.30. The titratable acidity of 'Regale' and 'Noble' juices stood out from the other varieties in both evaluated harvests due to their higher acidity levels. For the 2016 harvest only the juice from the 'Summit' variety reached a total soluble solids content of $14.00{ }^{\circ} \mathrm{Brix}$, the minimum content required for commercialization as a whole fruit juice according to Brazilian legislation. The fruits of this variety obtained the highest SS/TA ratio in the three harvests evaluated, with values close to 30 , an inverse behaviour to that of the 'Regale' juices, which presented the lowest averages for this variable, with values around 10. The red varieties gave rise to more acidic juices as compared to the white varieties. The 'Summit' variety presented the best sugar/acidity ratio, presenting aptitude for juice elaboration in the three harvests surveyed. The 'Regale' and 'Noble' varieties presented losses in productivity and sanitation in the 2016 harvest, although they were apt for juice production in the 2017 and 2018 harvests. The 'Carlos' variety juices did not reach the minimum content of $14^{\circ}$ Brix in any of the three harvests studied, and could not, therefore be classified as whole fruit juices.
\end{abstract}

Keywords: Muscadine; Processing; Composition; Quality; Harvest; Elaboration.

\title{
1 Introdução
}

As uvas Vitis rotundifolia, também denominadas uvas muscadíneas, pertencem à família Vitaceae, nativas do sudeste dos Estados Unidos. Atualmente, esse grupo compreende mais de cem cultivares (Pastrana-Bonilla et al., 2003; Wei et al. 2017), caracterizadas por apresentar cachos pequenos, bagas médias ou grandes, com coloração em geral marrom escuro, com variedades claras e bronzeadas. São pouco açucaradas, com muitos cachos por planta, poucas bagas por cacho, com formação de uma zona de abscisão entre a fruta e a ráquis. A casca é não estriada e aderente, de lenho duro e maturação escalonada (Sousa, 1996; Queiroz-Voltan \& Pires, 2003; Denega et al., 2010).

A produção de uvas muscadíneas é importante comercialmente no seu país de origem (EUA), em especial no estado da Georgia, onde apresenta grande popularidade, atribuída em parte aos atributos medicinais, nutricionais e terapêuticos, devido aos altos níveis de antioxidantes presentes nas bagas (Pastrana-Bonilla et al., 2003; Fonsah \& Awondo, 2016). Outro fator a ser considerado sobre estas uvas é a grande resistência à maioria das pragas e doenças da videira (Reisch et al., 2012), sendo atualmente empregadas mundialmente como fonte de resistência a doenças como o míldio e o oídio (Bouquet et al., 2000a), vírus (Bouquet et al., 2000b), nematoides de solo (Botton \& Colleta, 2010; Wei et al., 2017) e pérola-da-terra (Botton \& Colleta, 2010). No Brasil, são ainda pouco conhecidas e não possuem um mercado consumidor estabelecido (Giovanini, 2001; Sachi \& Biasi, 2008), entretanto trabalhos têm sido realizados no país com diferentes cultivares de uvas muscadíneas devido ao seu potencial para produção orgânica. Denega et al. (2010) avaliaram as características físicas e químicas dos cachos das variedades Topsail, Magnólia, Noble, Roanoke, Magoon, Regale, Bontiful e Dixie. Os autores concluíram que as uvas analisadas apresentaram baixo teor de sólidos solúveis e elevada acidez, não sendo recomendadas para o consumo in natura, porém com potencial para o processamento de sucos e geleias nas condições estudadas em Pinhais-PR. 
Park \& Oh (2015) relatam que as uvas da espécie Vitis rotundifolia possuem aroma e sabor bastante característicos, o que favorece o consumo in natura em relação ao suco ou vinho, no entanto, as uvas muscadíneas estão no mercado dos Estados Unidos há décadas, comercializadas como fruta fresca, sucos, geleias e vinhos (Fonsah \& Awondo, 2016).

Threlfall et al. (2007) avaliaram sucos produzidos a partir de oito cultivares de uvas muscadíneas quanto às características sensoriais, à composição físico-química e ao conteúdo nutricional. Os resultados demonstram que os maiores atributos descritivos verificados foram sabor doce, ácido e adstringente, e aroma de fruta cozida. Quanto à composição físico-química, ocorreram oscilações, sendo os sucos que apresentaram teor de sólidos solúveis igual a 14 e relação SS/AT de 26 a 31 melhor aceitos. Os autores alegam que mediante os parâmetros avaliados, as uvas muscadíneas podem gerar sucos de qualidade aceitável aos consumidores, com potencial nutracêutico, favorecendo a ampliação do mercado para estas.

Alexandrov \& Gaina (2015) alegam que as uvas destinadas à elaboração de suco podem conter um importante componente denominado antranilato de metila, composto responsável pelo aroma "foxado", que mantém no suco as características da uva in natura mesmo após o aquecimento e que é encontrado em uvas da espécie Vitis labrusca. As uvas muscadíneas, por sua vez, possuem um aroma bastante delicado, o qual pode ser prejudicado após o processamento a quente (Threlfall et al., 2005).

Os principais processos utilizados para elaboração do suco envolvem baixas temperaturas, nas quais as bagas são prensadas à temperatura ambiente, ou prensagem a quente, quando a uva é previamente aquecida e acrescida de enzimas pectolíticas para facilitar a extração do mosto (Guerra et al., 2016; Marcon et al., 2016). A extração a quente é tradicionalmente utilizada para sucos de uvas Vitis labrusca e, no caso de uvas Vitis rotundifolia, normalmente não se usa calor durante o processamento do suco, apesar de o aquecimento antes da prensagem favorecer o rendimento em suco, cor, sólidos solúveis e compostos fenólicos (Threlfall et al., 2007).

Diante do contexto apresentado, as uvas muscadíneas apresentam potencial para a diversificação da viticultura nacional, devido à importância para a produção orgânica; no entanto, são escassos os dados referentes aos produtos oriundos destas. Nesse sentido, este trabalho teve como objetivo avaliar sucos integrais obtidos a partir de quatro variedades de uvas da espécie Vitis rotundifolia quanto à composição físico-química.

\section{Material e métodos}

O trabalho foi realizado na Empresa de Pesquisa Agropecuária e Extensão Rural de Santa Catarina (Epagri), Estação Experimental de Videira (Videira, Santa Catarina, Brasil), localizada sob as coordenadas: $27^{\circ} 02^{\prime} 27,59^{\prime \prime} \mathrm{S}$ de latitude, $51^{\circ} 08^{\prime} 04,73^{\prime}$ ' W de longitude e altitude de 830 metros acima do nível do mar, durante as safras 2016, 2017 e 2018. O clima da região, de acordo com Köppen, é classificado como mesotérmico úmido e verão ameno (Cfb). As médias históricas (1960-2016) de precipitação, temperatura média e umidade relativa nos meses que englobam a brotação e colheita (setembro a março) são de $1.240 \mathrm{~mm}$, $19,8{ }^{\circ} \mathrm{C}$ e $73,4 \%$ UR, respectivamente (Empresa de Pesquisa Agropecuária e Extensão Rural de Santa Catarina, 2019).

As uvas empregadas na elaboração do suco foram da espécie Vitis rotundifolia, sendo duas tintas ('Regale' e 'Carlos') e duas brancas ('Summit' e 'Carlos'). O vinhedo foi implantado em 2000 no sistema de condução em latada sem o uso de porta-enxerto (pé franco), em espaçamento de 3,0 $\times 2,0 \mathrm{~m}$, entre linhas e entre plantas, respectivamente. A composição físico-química das bagas no momento da colheita das três safras avaliadas está listada na Tabela 1. 
Tabela 1. Aspectos físico-químicos das variedades de uva Vitis rotundifolia Regale, Noble, Summit e Carlos no momento da colheita, nas safras 2016, 2017 e 2018.

\begin{tabular}{cccccccccc}
\hline & & $\mathbf{p H}$ & \multicolumn{4}{c}{ Acidez titulável $\left(\mathbf{m E q . L ^ { - 1 } )}\right.$} & \multicolumn{3}{c}{ Sólidos Solúveis Totais $\left({ }^{\circ}\right.$ Brix) } \\
\hline & $\mathbf{2 0 1 6}$ & $\mathbf{2 0 1 7}$ & $\mathbf{2 0 1 8}$ & $\mathbf{2 0 1 6}$ & $\mathbf{2 0 1 7}$ & $\mathbf{2 0 1 8}$ & $\mathbf{2 0 1 6}$ & $\mathbf{2 0 1 7}$ & $\mathbf{2 0 1 8}$ \\
Regale & 3,18 & 3,31 & 3,00 & 116,06 & 83,92 & 78,33 & 12,80 & 15,20 & 14,56 \\
Noble & 3,50 & 3,29 & 3,03 & 94,01 & 71,55 & 59,89 & 13,10 & 15,50 & 15,86 \\
Summit & 3,47 & 3,43 & 3,36 & 48,28 & 52,31 & 39,60 & 15,90 & 16,20 & 16,86 \\
Carlos & 3,26 & 3,19 & 2,82 & 68,76 & 94,96 & 83,92 & 14,40 & 14,70 & 13,46 \\
\hline
\end{tabular}

As uvas foram colhidas nos meses de fevereiro e março de 2016, 2017 e 2018 manualmente e acondicionadas em caixas plásticas com capacidade para $20 \mathrm{~kg}$ de fruta até o momento do processamento.

As uvas brancas 'Summit' e 'Carlos' foram previamente desengaçadas e esmagadas mecanicamente; em seguida, a massa sólida foi levada à hidroprensa própria para pequenos volumes, na qual o mosto foi separado do bagaço. Foram adicionadas enzimas pectolíticas (Everzym XPL) e $\mathrm{SO}_{2}$ na forma de anidrido sulfuroso nas concentrações de $4 \mathrm{~mL} 100 \mathrm{~kg}^{-1}$ de uva e $20 \mathrm{mg} \mathrm{L}^{-1}$, respectivamente. Após a separação do líquido, este foi conduzido para a câmara fria a uma temperatura de $\pm 1{ }^{\circ} \mathrm{C}$ com adição de bentonite $\left(7 \mathrm{~mL} \mathrm{~L}^{-1}\right)$, para decantação das partículas sólidas por um período de 24 horas. No dia seguinte, o suco foi trasfegado, pasteurizado e engarrafado a uma temperatura de $86^{\circ} \mathrm{C}$.

Para as tintas 'Regale' e 'Noble', a extração do suco consistiu em desengace e esmagamento das bagas de forma mecânica, sendo, posteriormente, colocadas em recipiente de alumínio sobre fogareiro a gás, para o aquecimento até $50^{\circ} \mathrm{C}$. Adicionou-se complexo enzimático termorresistente comercial (Pectinex Ultra SP-L) na concentração de $3 \mathrm{~g} \mathrm{hL}^{-1}$. Após aproximadamente 20 minutos de constante homogeneização, o mosto, juntamente com o bagaço, atingiu a temperatura desejada, sendo retirado do fogo e mantido por cerca de 1 hora em maceração. Após o período de maceração, realizou-se a prensagem para separação do líquido, sendo este armazenado em câmara fria a uma temperatura de aproximadamente $1{ }^{\circ} \mathrm{C}$, para decantação das partículas sólidas, por 24 horas. No dia seguinte, o suco foi trasfegado, pasteurizado e engarrafado a uma temperatura de $86^{\circ} \mathrm{C}$.

Os sucos foram envazados em garrafas de vidro transparente com capacidade de $500 \mathrm{~mL}$, mantidas em temperatura ambiente em local próprio para armazenamento de bebidas (livre de odores e luminosidade excessiva), até o momento da execução das análises físico-químicas.

O experimento contou com delineamento inteiramente casualizado, com três repetições, em que cada repetição foi representada por uma garrafa, escolhidas aleatoriamente para as análises físico-químicas (realizadas em triplicata), em esquema bifatorial $(4 \times 3)$, sendo o fator A, as variedades (Regale, Noble, Summit e Carlos), e o fator B, as safras (2016, 2017 e 2018).

As análises físico-químicas de acidez total (AT), teor de sólidos solúveis (SS), relação SS/AT e pH foram realizadas nas uvas antes do processamento, bem como no suco finalizado, seguindo Instrução Normativa n. ${ }^{\circ} 24$ de 08/09/2005 (Brasil, 2005). O teor de sólidos solúveis foi determinado em refratômetro digital de bancada com compensação automática de temperatura (QUIMIS ${ }^{\circledR}$ ) e o resultado expresso em ${ }^{\circ}$ Brix. $\mathrm{O}$ pH foi determinado em pHmetro Meter AD1030 e as determinações de acidez total foram realizadas por titulação da amostra, com solução padronizada de $\mathrm{NaOH} 0,1 \mathrm{~N}$, adotando-se, como ponto final da titulação, o $\mathrm{Ph}=8,2$, sendo o resultado expresso em $\mathrm{mEq} \mathrm{L} \mathrm{L}^{-1}$. A relação SS/AT foi determinada pela obtenção do quociente da divisão entre os sólidos solúveis ( ${ }^{\circ}$ Brix) e acidez titulável em g $100 \mathrm{~mL}^{-1}$ de ácido tartárico.

Os sucos foram avaliados ainda quanto aos açúcares redutores totais $\left(\mathrm{g} \mathrm{L}^{-1}\right)$ utilizando o método DNS descrito por Maldonade et al. (2016), adaptado para suco de uva. A densidade foi determinada com um densímetro de vidro Aton Paar, expressa $\mathrm{em} \mathrm{g} \mathrm{cm}^{-3}$. Para avaliar a coloração dos sucos, foi empregado o espectrofotômetro da Konica Minolta, modelo CM-5, pesquisando as coordenadas L*, $\mathrm{a}^{*}$ e $\mathrm{b}^{*}$, sendo que o $\mathrm{L}^{*}$ representa a luminosidade da amostra e os valores de $\mathrm{a}^{*} \mathrm{e} \mathrm{b}^{*}$ foram empregados nos cálculos da saturação (croma) e tonalidade $\left({ }^{\circ} \mathrm{HUE}\right)$ da cor, 
obtidas pelas seguintes fórmulas: $\mathrm{C}^{*}=\left[\left(\mathrm{a}^{*}\right)^{2}+\left(\mathrm{b}^{*}\right)^{2}\right]^{1 / 4}$ e $\mathrm{H}^{0}=\tan ^{-1}\left(\mathrm{~b}^{*} / \mathrm{a}^{*}\right)$, quando $\mathrm{a}^{*}>0$ e $\mathrm{b}^{*} \geq 0$ $\mathrm{H}^{\mathrm{o}}=180+\tan ^{-1}\left(\mathrm{~b}^{*} / \mathrm{a}^{*}\right)$ e quando $\mathrm{a}^{*}<0$, respectivamente, conforme recomendações de McGuire (1992).

Os dados foram submetidos à análise de variância (ANOVA) e, quando detectados efeitos de tratamento, procedeu-se ao teste de comparação de médias pelo Teste Tukey a 5\% de probabilidade de erro.

\section{Resultados e discussão}

Os resultados obtidos apresentaram interação entre os fatores de tratamento avaliados (safras $\times$ variedades) pelo Teste Tukey a 5\% de probabilidade de erro, sendo estes listados nas Tabelas 2, 3 e 4 . Os dados climáticos correspondentes ao período de maturação das uvas estão listados na Tabela 5.

Foram verificadas diferenças significativas dos sucos para o $\mathrm{pH}$ entre as safras dentro de cada variedade, exceto para a 'Carlos', nas safras 2016 e 2017. Para o fator safra, ocorreu diferença para todas as variedades testadas (Tabela 2). 'Regale' e 'Carlos' apresentaram valores que se mantiveram na faixa de 2,82 a 2,98, e para 'Noble' e 'Summit', os valores foram superiores, chegando a 3,30. Rizzon \& Meneguzzo (2007) alegam que quanto menor o valor do $\mathrm{pH}$ mais fácil se torna a destruição térmica dos microrganismos, sendo que o valor mínimo do $\mathrm{pH}$ em sucos de uva varia de 3,00 a 3,10, valores superiores aos verificados no presente estudo para as variedades 'Regale' e 'Carlos'. Segundo Santana et al. (2008), o pH está relacionado às características gustativas dos sucos e pode ser influenciado principalmente pela característica genética das diferentes cultivares utilizadas e pelo processamento. Threlfall et al. (2005) avaliaram sucos de Black Beauty (Vitis rotundifolia) e Sunbelt (Vitis labrusca) elaborados com prensagem a quente e à temperatura ambiente, obtendo valores de 3,27 e 3,45 para 'Black Beauty', e 3,42 e 3,48 para 'Sunbelt'. Threlfall et al. (2007) encontraram valores de 3,32 para sucos de 'Summit' e 3,09 para 'Carlos', em trabalho avaliando oito variedades de uvas muscadíneas. Toaldo et al. (2015) avaliaram sucos de uvas Vitis labrusca produzidas de maneira convencional e orgânica, obtendo valores que variaram de 3,03 até 3,23.

Tabela 2. Valores de pH, acidez titulável e sólidos solúveis totais de sucos elaborados a partir das uvas Vitis rotundifolia Regale, Noble, Summit e Carlos, nas safras 2016, 2017 e 2018.

\begin{tabular}{|c|c|c|c|c|c|c|}
\hline \multirow{4}{*}{$\begin{array}{c}\text { Variedades } \\
\text { Regale }\end{array}$} & \multicolumn{6}{|c|}{ Variáveis } \\
\hline & \multicolumn{6}{|c|}{$\mathrm{pH}$} \\
\hline & \multicolumn{2}{|c|}{2016} & \multicolumn{2}{|c|}{2017} & \multicolumn{2}{|c|}{2018} \\
\hline & 2,82 & $\mathrm{cD}^{1 *}$ & 2,98 & $\mathrm{aC}$ & 2,93 & $\mathrm{bC}$ \\
\hline Noble & 3,03 & $\mathrm{cB}$ & 3,19 & $\mathrm{aB}$ & 3,06 & $\mathrm{bB}$ \\
\hline Summit & 3,19 & $\mathrm{cA}$ & 3,30 & $\mathrm{aA}$ & 3,22 & $\mathrm{bA}$ \\
\hline \multirow[t]{3}{*}{ Carlos } & 2,95 & $\mathrm{aC}$ & 2,93 & $\mathrm{aD}$ & 2,89 & $\mathrm{bD}$ \\
\hline & \multicolumn{6}{|c|}{ Acidez Titulável $\left(\mathrm{mEq} \mathrm{L}^{-1}\right)$} \\
\hline & \multicolumn{2}{|c|}{2016} & \multicolumn{2}{|c|}{2017} & \multicolumn{2}{|c|}{2018} \\
\hline Regale & 168,73 & $\mathrm{aA}$ & 135,44 & $\mathrm{cA}$ & 160,10 & $\mathrm{bA}$ \\
\hline Noble & 121,68 & $\mathrm{aB}$ & 96,90 & $\mathrm{bB}$ & 120,10 & $\mathrm{aB}$ \\
\hline Summit & 70,00 & $\mathrm{aD}$ & 55,95 & $\mathrm{cC}$ & 61,23 & $\mathrm{bD}$ \\
\hline \multirow[t]{3}{*}{ Carlos } & 99,40 & $\mathrm{aC}$ & 97,80 & $\mathrm{abB}$ & 94,63 & $\mathrm{bC}$ \\
\hline & \multicolumn{6}{|c|}{ Sólidos Solúveis Totais $\left({ }^{\circ}\right.$ Brix $)$} \\
\hline & \multicolumn{2}{|c|}{2016} & \multicolumn{2}{|c|}{2017} & \multicolumn{2}{|c|}{2018} \\
\hline Regale & 12,00 & $\mathrm{bD}$ & 14,33 & $\mathrm{aB}$ & 14,30 & $\mathrm{aB}$ \\
\hline Noble & 12,30 & $\mathrm{cC}$ & 14,17 & $\mathrm{bB}$ & 15,70 & $\mathrm{aA}$ \\
\hline Summit & 14,40 & $\mathrm{bA}$ & 15,23 & $\mathrm{aA}$ & 15,47 & $\mathrm{aA}$ \\
\hline Carlos & 13,07 & $\mathrm{aB}$ & 11,90 & $\mathrm{bC}$ & 12,97 & $\mathrm{aC}$ \\
\hline
\end{tabular}

${ }^{1}$ Mesma letra maiúscula na coluna não difere entre variedades dentro de safras. *Mesma letra minúscula na linha não difere entre safras dentro de variedades.

A acidez titulável dos sucos de 'Regale' e 'Noble' se destacou significativamente das demais variedades em ambas as safras avaliadas, devido aos valores mais elevados. Os sucos de 'Summit' demonstraram uma menor acidez, seguidos dos sucos de 'Carlos'. As uvas colhidas na safra de 2016 originaram sucos mais 
ácidos, enquanto que, nos sucos produzidos a partir de uvas da safra de 2017, observou-se uma queda nos teores. Em 2018, os valores foram um pouco mais elevados em relação à safra anterior (Tabela 2). A safra de 2016 foi prejudicada devido a eventos climáticos ocorridos na região, dentre os quais, o excesso de precipitação no período de maturação das uvas, conforme dados descritos na Tabela 5. Já na safra 2017, as chuvas foram inferiores, e em 2018, o volume foi intermediário. O maior volume de chuvas e dias nublados, bem como menor insolação, dificultam a degradação dos ácidos presentes nas bagas ao longo da maturação e este fator pode ser o responsável pela maior acidez observada nos sucos da safra 2016. A diferença na acidez entre variedades brancas e tintas pode ser explicada levando em consideração os resultados de Sachi \& Biasi (2008), que avaliaram a evolução da maturação de quatro variedades de uvas muscadíneas em Pinhais-PR, as brancas 'Dixie' e 'Roanok', e as tintas 'Regale' e Bontiful'. Esses autores verificaram que as uvas brancas apresentaram menores teores de acidez, em comparação às tintas. Outro fator que também pode ter influenciado na acidez dos sucos tintos é o fato de terem passado por aquecimento e um período de maceração antes da prensagem. Threlfall et al. (2005) alegam que a extração a quente favorece o incremento de ácidos nos sucos, devido à plasmólise da membrana celular, que facilita a extração dos ácidos das cascas e sementes (Lima et al., 2015; Mota et al., 2018).

Para o teor de sólidos solúveis totais, na safra 2016, apenas os sucos da variedade Summit atingiram o valor de 14,00 ํix, teor mínimo exigido para comercialização como suco integral perante a legislação brasileira (Brasil, 2018) (Tabela 2). Cabe ressaltar que a safra de 2016 foi extremamente atípica, devido a problemas climáticos nos estados da região Sul do Brasil, resultando em prejuízos significativos na produção e qualidade das uvas (Mello \& Machado, 2018; Mello, 2018). Nas safras 2017 e 2018, o teor mínimo de sólidos solúveis totais exigido em lei foi superado pelos sucos de 'Regale', 'Noble' e 'Summit', sendo o maior valor de 15,70 'Brix para 'Noble', em 2018. Para 'Carlos', os valores se mantiveram $13,07,11,90$ e $12,97^{\circ}$ Brix, respectivamente, para as safras 2016 , 2017 e 2018. De acordo com Robaskewicz et al. (2016), o teor de sólidos solúveis totais indica, aproximadamente, a quantidade de açúcar existente no fruto e que é transferido ao suco. Threlfall et al. (2005) afirmam que a prensagem da uva quente favorece a extração dos sólidos solúveis para o suco e esta afirmação corrobora com os resultados observados nas amostras das safras 2017 e 2018, uma vez que os sucos das variedades tintas que foram prensados quentes apresentaram valores muito próximos aos encontrados nas uvas antes do processamento, enquanto que, nos sucos brancos, os valores foram inferiores. Denega et al. (2010) avaliaram as características físicas e químicas dos cachos de oito variedades de uvas Vitis rotundifolias, em Pinhais-PR, nas safras 2004 e 2005, e os resultados demonstram que os teores de sólidos solúveis totais variaram de 12,7 a 13,7 ${ }^{\circ}$ Brix para 'Noble', e foram 9,2 e 8,9 'Brix para 'Regale', em 2004 e 2005, respectivamente. Estes autores concluíram que as uvas muscadíneas apresentam baixo teor de sólidos solúveis.

Os sucos da variedade Summit obtiveram a maior relação SS/AT nas três safras avaliadas, com valores próximos a 30, comportamento diverso do verificado nos sucos de 'Regale', que apresentaram as menores médias para esta variável, com valores em torno de 10. As demais variedades obtiveram valores acima de 15 , exceto os sucos de 'Noble', em $2016(13,48)$ (Tabela 3). A relação SS/AT representa o equilíbrio entre o gosto doce e o gosto ácido dos sucos, sendo, portanto, um indicativo de qualidade dos mesmos (Gurak et al., 2012). Rizzon \& Meneguzzo (2007) dizem que os valores para esta relação devem estar entre 15 e 45. Lopes et al. (2017) explicam que valores baixos representam um desequilíbrio entre o gosto doce e o gosto ácido do suco de uva, o que pode ser usado como indicativo de queda da qualidade, quando tal relação é utilizada em sucos de variedades labruscas. No caso de muscadíneas, essa relação mais baixa é característica do produto e pode ser apreciada pelos consumidores. Sachi \& Biasi (2008) e Denega et al. (2010) descrevem as uvas muscadíneas como pouco açucaradas e bastante ácidas, fator que as prejudica quanto ao equilíbrio gustativo. 
Tabela 3. Valores de relação SS/AT, açúcares redutores totais e densidade de sucos elaborados a partir das uvas Vitis rotundifolia Regale, Noble, Summit e Carlos, nas safras 2016, 2017 e 2018.

\begin{tabular}{|c|c|c|c|c|c|c|}
\hline \multirow{3}{*}{ Variedades } & \multicolumn{6}{|c|}{ Variáveis } \\
\hline & \multicolumn{6}{|c|}{ Relação SS/AT } \\
\hline & \multicolumn{2}{|c|}{2016} & \multicolumn{2}{|c|}{2017} & \multicolumn{2}{|c|}{2018} \\
\hline Regale & 9,48 & $\mathrm{cD}^{1 *}$ & 14,12 & $\mathrm{aD}$ & 11,91 & $\mathrm{bC}$ \\
\hline Noble & 13,48 & $\mathrm{cC}$ & 19,50 & $\mathrm{aB}$ & 17,43 & $\mathrm{bB}$ \\
\hline Summit & 27,43 & $\mathrm{cA}$ & 36,38 & $\mathrm{aA}$ & 33,69 & $\mathrm{bA}$ \\
\hline \multirow[t]{3}{*}{ Carlos } & 17,53 & $\mathrm{abB}$ & 16,23 & $\mathrm{bC}$ & 18,27 & $\mathrm{aB}$ \\
\hline & \multicolumn{6}{|c|}{ Açúcares Redutores Totais $\left(\mathrm{g} \mathrm{L}^{-1}\right)$} \\
\hline & \multicolumn{2}{|c|}{2016} & \multicolumn{2}{|c|}{2017} & \multicolumn{2}{|c|}{2018} \\
\hline Regale & 131,17 & $\mathrm{bC}$ & 161,83 & $\mathrm{aA}$ & 152,93 & $\mathrm{aA}$ \\
\hline Noble & 137,00 & $\mathrm{bC}$ & 153,67 & $\mathrm{aA}$ & 152,70 & $\mathrm{aA}$ \\
\hline Summit & 166,17 & $\mathrm{aA}$ & 162,83 & $\mathrm{aA}$ & 121,60 & $\mathrm{bB}$ \\
\hline \multirow[t]{3}{*}{ Carlos } & 151,33 & $\mathrm{aB}$ & 130,83 & $\mathrm{bB}$ & 123,10 & $\mathrm{bB}$ \\
\hline & \multicolumn{6}{|c|}{ Densidade $\left(\mathrm{g} \mathrm{L}^{-1}\right)$} \\
\hline & \multicolumn{2}{|c|}{2016} & \multicolumn{2}{|c|}{2017} & \multicolumn{2}{|c|}{2018} \\
\hline Regale & 1052 & $\mathrm{bC}$ & 1061 & $\mathrm{aB}$ & 1061 & $\mathrm{aC}$ \\
\hline Noble & 1053 & $\mathrm{cC}$ & 1061 & $\mathrm{bB}$ & 1062 & $\mathrm{aB}$ \\
\hline Summit & 1060 & $\mathrm{cA}$ & 1065 & $\mathrm{bA}$ & 1067 & $\mathrm{aA}$ \\
\hline Carlos & 1055 & $\mathrm{bB}$ & 1051 & $\mathrm{cC}$ & 1057 & $\mathrm{aD}$ \\
\hline
\end{tabular}

${ }^{1}$ Mesma letra maiúscula na coluna não difere entre variedades dentro de safras. *Mesma letra minúscula na linha não difere entre safras dentro de variedades.

As variedades brancas apresentaram maiores valores para açúcares redutores totais na safra 2016, enquanto que, na safra 2018, as variedades tintas se destacaram. Na safra 2017, os sucos da variedade Carlos diferiram dos demais devido ao menor teor de açúcares (Tabela 3). O suco de uva possui elevado teor de açúcares (glicose e frutose) e estes valores podem variar de 15\% a 30\% em função de alguns fatores, como o clima, o solo, o estádio de maturação e a variedade (Carneiro et al., 2013). As variedades muscadíneas apresentam um ciclo mais tardio que as Vitis labrusca tradicionalmente empregadas na elaboração do suco, com a maturação transcorrendo em meados de fevereiro e se estendendo a março até início de abril, ou seja, ocorre no período chuvoso na região, de acordo com dados da EPAGRI/CIRAM. Estes demonstram que os maiores volumes de chuva na safra 2016 foram nos meses de fevereiro e março, e em 2017, os volumes se mantiveram bastante próximos em janeiro, fevereiro e março, enquanto que março de 2018 se mostrou o mais chuvoso (Tabela 5). Os volumes diferenciados de chuva entre as safras estudadas podem ser responsáveis pelas diferenças observadas no teor de açúcares redutores nos sucos. Fongaro et al. (2016) estudaram a evolução físico-química de sucos brancos e tintos produzidos na Serra Gaúcha dentre os anos de 2012 a 2016, encontrando diferença significativa na safra 2014 entre os sucos brancos e tintos. Os autores alegaram que a diferença pode ser devida à possibilidade de as uvas terem sido originadas de diferentes locais, com solos de diferentes composições, sob diferentes tratamentos tecnológicos da videira ao produto final. Mota et al. (2018) avaliaram sucos de uvas americanas e híbridas produzidas no estado de Minas Gerais nas safras 2013 e 2014, encontrando valores que variaram de $98,5 \mathrm{~g} \mathrm{~L}^{-1}$ a $127,6 \mathrm{~g} \mathrm{~L}^{-1}$, inferiores aos do presente estudo.

Os sucos da variedade 'Summit' apresentaram maior densidade em relação aos demais nas três safras 2016, 2017 e 2018 (Tabela 3). Silva et al. (2011) verificaram valores de $1.066 \mathrm{~g} \mathrm{~L}^{-1}$ para sucos da variedade BRS Violeta e $1.062 \mathrm{~g} \mathrm{~L}^{-1}$ para sucos de Isabel Precoce, e os autores associaram essa diferença com a porcentagem de sólidos solúveis presentes nos sucos elaborados, os quais apresentaram valores de 19,1 e 14,8, expressos em ${ }^{\circ}$ Brix, para as variedades BRS Violeta e Isabel Precoce, respectivamente. Rizzon \& Link (2006) encontraram uma média de $1.055 \mathrm{~g} \mathrm{~L}^{-1}$ para sucos das variedades Isabel, Bordô, Concord e Cabernet Sauvignon elaborados pelo sistema de arraraste a vapor.

Para as variáveis de cor, os sucos das variedades tintas apresentaram valores menores para luminosidade na safra 2018 em relação aos das safras anteriores, com uma tonalidade na faixa do vermelho púrpura, enquanto que, nas safras 2016 e 2017, o ângulo de nuances da cor indica que os sucos de Regale e Noble estavam na faixa do 
laranja avermelhado em 2016 e do vermelho em 2017 (Tabela 4). Para os sucos das uvas brancas, a luminosidade manteve-se acima de 90 para as duas variedades, indicando sucos bastante claros, com tonalidade na faixa do amarelo ao amarelo esverdeado. A maior intensidade de cor foi verificada nos sucos tintos na safra 2018, diverso aos brancos, que apresentaram a menor intensidade nesta mesma safra (Tabela 4). As diferenças de cor verificadas entre as safras podem indicar um início de oxidação dos sucos produzidos em 2016, fator que pode ter sido desencadeado pelo estado sanitário das uvas no momento da colheita. Threlfall et al. (2007) encontraram valores de 25,8 a 53,4 para a tonalidade dos sucos das variedades muscadíneas tintas, indicando que a coloração variou do vermelho púrpura ao laranja, e para os sucos das variedades brancas, os valores foram 98,4 a 99,5, próximos aos verificados nos sucos de 'Summit' e 'Carlos' analisados neste estudo.

Tabela 4. Valores de luminosidade, tonalidade e intensidade da cor de sucos elaborados a partir das uvas Vitis rotundifolia Regale, Noble, Summit e Carlos, nas safras 2016, 2017 e 2018.

\begin{tabular}{|c|c|c|c|c|c|c|}
\hline \multirow{3}{*}{ Variedades } & \multicolumn{6}{|c|}{ Variáveis } \\
\hline & \multicolumn{6}{|c|}{ Luminosidade (*L) } \\
\hline & \multicolumn{2}{|c|}{2016} & \multicolumn{2}{|c|}{2017} & \multicolumn{2}{|c|}{2018} \\
\hline Regale & 80,33 & $\mathrm{aC}^{1 *}$ & 80,28 & $\mathrm{aC}$ & 72,11 & $\mathrm{bB}$ \\
\hline Noble & 81,64 & $\mathrm{bB}$ & 82,59 & $\mathrm{aB}$ & 55,46 & $\mathrm{cC}$ \\
\hline Summit & 95,20 & $\mathrm{bA}$ & 95,94 & $\mathrm{aA}$ & 96,30 & $\mathrm{aA}$ \\
\hline \multirow[t]{3}{*}{ Carlos } & 95,65 & $\mathrm{bA}$ & 96,07 & $\mathrm{bA}$ & 96,74 & $\mathrm{aA}$ \\
\hline & \multicolumn{6}{|c|}{ Tonalidade de Cor ( $\left.{ }^{\circ} \mathrm{HUE}\right)$} \\
\hline & \multicolumn{2}{|c|}{2016} & \multicolumn{2}{|c|}{2017} & \multicolumn{2}{|c|}{2018} \\
\hline Regale & 65,71 & $\mathrm{aB}$ & 31,12 & $\mathrm{bB}$ & 6,36 & $\mathrm{cD}$ \\
\hline Noble & 58,33 & $\mathrm{bC}$ & 32,45 & $\mathrm{cB}$ & 356,37 & $\mathrm{aA}$ \\
\hline Summit & 92,61 & $\mathrm{aA}$ & 92,73 & $\mathrm{aA}$ & 85,42 & $\mathrm{bC}$ \\
\hline \multirow[t]{3}{*}{ Carlos } & 93,68 & $\mathrm{aA}$ & 90,15 & $\mathrm{bA}$ & 90,79 & $\mathrm{bB}$ \\
\hline & \multicolumn{6}{|c|}{ Intensidade da Cor (Croma) } \\
\hline & \multicolumn{2}{|c|}{2016} & \multicolumn{2}{|c|}{2017} & \multicolumn{2}{|c|}{2018} \\
\hline Regale & 28,86 & $\mathrm{bA}$ & 22,34 & $\mathrm{cA}$ & 37,02 & $\mathrm{aB}$ \\
\hline Noble & 23,04 & $\mathrm{bB}$ & 18,93 & $\mathrm{cB}$ & 58,60 & $\mathrm{aA}$ \\
\hline Summit & 6,04 & $\mathrm{aC}$ & 3,38 & $\mathrm{bC}$ & 1,13 & $\mathrm{cC}$ \\
\hline Carlos & 4,57 & $\mathrm{aD}$ & 2,65 & $\mathrm{bC}$ & 0,73 & $\mathrm{cC}$ \\
\hline
\end{tabular}

${ }^{1}$ Mesma letra maiúscula na coluna não difere entre variedades dentro de safras. *Mesma letra minúscula na linha não difere entre safras dentro de variedades.

Tabela 5. Dados climáticos referentes ao período de maturação das variedades de uva Vitis rotundifolia Regale, Noble, Summit e Carlos, nas safras 2016, 2017 e 2018.

\begin{tabular}{|c|c|c|c|c|c|}
\hline \multirow{3}{*}{ Meses } & \multicolumn{5}{|c|}{ Dados Climáticos } \\
\hline & \multicolumn{5}{|c|}{ Safra 2016} \\
\hline & $\begin{array}{c}\text { Temp. Mínima } \\
\left({ }^{\circ} \mathrm{C}\right)\end{array}$ & $\begin{array}{c}\text { Temp. Máxima } \\
\left({ }^{\circ} \mathrm{C}\right)\end{array}$ & $\begin{array}{c}\text { Temp. Média } \\
\left({ }^{\circ} \mathrm{C}\right)\end{array}$ & $\begin{array}{c}\text { Ampl. Térmica } \\
\left({ }^{\circ} \mathrm{C}\right)\end{array}$ & $\begin{array}{l}\text { Precipitação } \\
\text { (mm) }\end{array}$ \\
\hline Janeiro & 13,40 & 34,40 & 23,90 & 21,00 & 144,20 \\
\hline Fevereiro & 15,00 & 33,00 & 24,00 & 18,00 & 226,30 \\
\hline Março & 11,20 & 31.20 & 21.20 & 20,00 & 259,70 \\
\hline \multirow[b]{2}{*}{ Meses } & \multicolumn{5}{|c|}{ Safra 2017} \\
\hline & $\begin{array}{c}\text { Temp. Mínima } \\
\left({ }^{\circ} \mathrm{C}\right)\end{array}$ & $\begin{array}{c}\text { Temp. Máxima } \\
\left({ }^{\circ} \mathrm{C}\right)\end{array}$ & $\begin{array}{c}\text { Temp. Média } \\
\left({ }^{\circ} \mathrm{C}\right)\end{array}$ & $\begin{array}{c}\text { Ampl. Térmica } \\
\left({ }^{\circ} \mathrm{C}\right)\end{array}$ & $\begin{array}{c}\text { Precipitação } \\
\text { (mm) }\end{array}$ \\
\hline Janeiro & 13,65 & 32,56 & 22,5 & 18,91 & 151,00 \\
\hline Fevereiro & 14,59 & 32,56 & 22,6 & 17,97 & 157,80 \\
\hline Março & 10,86 & 31,54 & 20,6 & 20,68 & 103,60 \\
\hline \multirow[b]{2}{*}{ Meses } & \multicolumn{5}{|c|}{ Safra 2018} \\
\hline & $\begin{array}{c}\text { Temp. Mínima } \\
\left({ }^{\circ} \mathrm{C}\right)\end{array}$ & $\begin{array}{c}\text { Temp. Máxima } \\
\left({ }^{\circ} \mathrm{C}\right)\end{array}$ & $\begin{array}{c}\text { Temp. Média } \\
\left({ }^{\circ} \mathrm{C}\right)\end{array}$ & $\begin{array}{c}\text { Ampl. Térmica } \\
\left({ }^{\circ} \mathrm{C}\right)\end{array}$ & $\begin{array}{l}\text { Precipitação } \\
\text { (mm) }\end{array}$ \\
\hline Janeiro & 17,53 & 28,02 & 21,55 & 10,49 & 47,28 \\
\hline Fevereiro & 15,51 & 26,93 & 20,54 & 11,42 & 190,05 \\
\hline Março & 16,94 & 27,78 & 21,24 & 10,84 & 218,6 \\
\hline
\end{tabular}

Fonte: Empresa de Pesquisa Agropecuária e Extensão Rural de Santa Catarina (2019). 


\section{Conclusões}

As variedades tintas originaram sucos mais ácidos em comparação às variedades brancas.

Os sucos elaborados de uvas muscadíneas da variedade Carlos apresentaram baixo teor de sólidos solúveis nas três safras avaliadas.

A variedade 'Summit' apresentou a melhor relação açúcar/acidez devido ao maior teor de sólidos solúveis e menor acidez titulável, quando comparada às demais variedades testadas, apresentando aptidão para a elaboração de sucos nas três safras pesquisadas.

\section{Referências}

Alexandrov, E., \& Gaina, B. (2015). Distant hybrids in F4 (Vitis vinifera L. x Muscadinia rotundifolia michx.) and of cultivars of Vitis vinifera L. and of concerning the content of some biochemical compounds. Scientific Papers. Series Management, Economic, Engineering in Agriculture and Rural Development, 15(1), 37-44.

Botton, M., \& Colleta, V. D. (2010). Avaliação da resistência de cultivares de Vitis rotundifolia à pérolada-terra (Hemiptera: Margarodidae) na região sul do Brasil. Acta Scientiarum: Agronomy, 32(2), 213-216. http://dx.doi.org/10.4025/actasciagron.v32i2.3151

Bouquet, A., Danglot, Y., Bongiovanni, M., Castagnone-Sereno, P., Esmenjaud, D., Dalmasso, A., \& Torregrosa, L. (2000b). Breeding rootstocks resistant to grape fanleaf virus spread using Vitis $\times$ Muscadinia hybridization. Acta Horticulturae, (528), 517-526. http://dx.doi.org/10.17660/ActaHortic.2000.528.75

Bouquet, A., Pauquet, J., Adam-Blondon, A., Torregrosa, L., Merdinoglu, L., \& Wiedemann-Merdinoglu, D. (2000a). Vers l'obtention de variétés de vigne résistantes à l'oïdium et au mildiou par les méthodes conventionnelles et biotechnologiques. Bulletin de l'OIV, (73), 445-452.

Brasil. Ministério da Agricultura, Pecuária e Abastecimento. (2005, 20 de setembro). Aprova o Manual Operacional de Bebidas e Vinagre (Instrução normativa $n^{\circ} 24$, de 8 de setembro de 2005). Diário Oficial da União, Brasília.

Brasil. Ministério da Saúde. Secretaria de Vigilância Sanitária. (2018, 9 de março). Complementação dos padrões de identidade e qualidade do vinho e dos derivados da uva e do vinho (Instrução Normativa $n^{\circ} 14$, de 8 de fevereiro de 2018). Diário Oficial da União, Brasília, seção 1.

Carneiro, A. P. G., Abreu, D. A., Soares, D. J., Costa, E. A., Silva, L. M. R., Barbosa, L. C., Sousa, P. H. M., \& Figueiredo, R. W. (2013). Avaliação da rotulagem, caracterização química, físico-química e reológica de néctares de uva comercializados na cidade de Fortaleza - CE. Brazilian Journal of Food and Nutrition, 24(2), 241-249.

Denega, S., Biasi, L. A., Zanette, F., Maggi, M. F., Jadoski, S. O., \& Blaskevicz, S. J. (2010). Características físicas e químicas dos cachos de cultivares de Vitis rotundifolia. Semina. Ciências Agrárias, 31(3), 633-638. http://dx.doi.org/10.5433/16790359.2010v31n3p633

Empresa de Pesquisa Agropecuária e Extensão Rural de Santa Catarina - EPAGRI. Centro de Informações de Recursos Ambientais e de Hidrometeorologia de Santa Catarina - CIRAM. (2019). Dados climáticos. Florianopolis. . Recuperado em 1 de novembro de 2019, de http://ciram.epagri.sc.gov.br/

Fongaro, C., Cavagnolli, N. I., \& Spada, P. K. W. D. S. (2016). Evaluation of physicochemical parameters of grape juices produced in the Serra Gaúcha. BIO Web of Conferences, 7, 01008. http://dx.doi.org/10.1051/bioconf/20160701008

Fonsah, E. G., \& Awondo, S. N. (2016). Cost estimates and investment analysis for muscadine grapes production in Georgia. Journal of Food Distribution Research, 47(1), 1-5.

Giovanini, E. (2001). Uva agroecológica (1. ed.). Porto Alegre: Renascença.

Guerra, C. C., Bitarelo, H., \& Ben, R. L. (2016). Sistema para elaboração de suco de uva integral em pequenos volumes: Suquificador integral (Documentos, 96, p. 32). Bento Gonçalves: Embrapa.

Gurak, P. D., Silva, M. C., Matta, V. M., Rocha-Leão, M. H., \& Cabra, L. M. C. (2012). Avaliação de parâmetros físico-químicos de sucos de uva integral, néctares de uva e néctares de uva light. Revista de Ciências Exatas, 27-31(1), 7-22.

Lima, M. S., Dutra, M. C. P., Toaldo, I. M., Corrêa, L. C., Pereira, G. E., Oliveira, D., Bordignon-Luiz, M. T., \& Ninow, J. L. (2015). Phenolic compounds, organic acids and antioxidant activity of grape juices produced in industrial scale by different processes of maceration. Food Chemistry, 188, 384-392. PMid:26041208. http://dx.doi.org/10.1016/j.foodchem.2015.04.014

Lopes, I. A., Silva, J. R., Lima, L. T., Santos, V. L. V., \& Silva, S. P. (2017). Análises físico-químicas em sucos de uva: Integral, reprocessado, concentrado e desidratado comercializados em Garanhuns-PE. Revista Brasileira de Agrotecnologia, 7(2), 4548.

Maldonade, I. R., Carvalho, P. G. B., \& Ferreira, N. A. (2016). Protocolo para determinação de açúcares totais em hortaliças pelo método DNS (Documentos, No. 96, pp. 32). Brasília: Embrapa.

Marcon, Â. R., Dutra, S. V., Roani, C. A., Spinelli, F. R., Leonardelli, S., Venturin, L., \& Vanderlinde, R. (2016). Avaliação da incorporação de água exógena em sucos de uva elaborados por panela extratora. Revista Brasileira de Viticultura e Enologia, (8), 52-57.

McGuire, R. G. (1992). Reporting of objective color measurements. HortScience, 27(12), 1254-1255.

http://dx.doi.org/10.21273/HORTSCI.27.12.1254 
Mello, L. M. R. (2018). Desempenho da vitivinicultura brasileira em 2017 (Anuário Campo e Negócio). Brasília: Embrapa.

Mello, L. M. R., \& Machado, C. A. E. (2018). Dados da viticultura. Brasília: Embrapa. Recuperado em 15 de setembro de 2018, de http://vitibrasil.cnpuv.embrapa.br/

Mota, R. V., Glória, M. B. A., Souza, B. S., Peregrino, I., Pimentel, R. M. A., Dias, F. A. N., Souza, L. C., Souza, A. L., \& Regina, M. A. (2018). Bioactive compounds and juice quality from selected grape cultivars. Bragantia, 77(1), 62-73. http://dx.doi.org/10.1590/1678-4499.2016369

Park, M., \& Oh, J. (2015). Antioxidant and antimicrobial activities of muscadine grape extracts. The Korean Society of Food Preservation, 21(1), 12-18. http://dx.doi.org/10.11002/kjfp.2015.22.1.12

Pastrana-Bonilla, E., Akoh, C. C., Sellappan, S., \& Krewer, G. (2003). Phenolic content and antioxidant capacity of muscadine grapes. Journal of Agricultural and Food Chemistry, 51(18), 5497-5503. PMid:12926904. http://dx.doi.org/10.1021/jf030113c Queiroz-Voltan, R. B., \& Pires, E. J. P. (2003). A videira. In C. V. Pommer (Ed.), Uva: Tecnologia de produção, pós colheita, mercado (pp. 37-61). Porto Alegre: Cinco Continentes.

Reisch, B. I., Owens, C. L., \& Cousins, P. S. (2012). Grape. In M. Badenes \& D. Byrne (Eds.), Fruit breeding (Handbook of Plant Breeding, No. 8). Boston: Springer.

Rizzon, L. A., \& Link, M. (2006). Composição do suco de uva caseiro de diferentes cultivares. Ciência Rural, Santa Maria, 36(2), 689-692. http://dx.doi.org/10.1590/S0103-84782006000200055

Rizzon, L. A., \& Meneguzzo, J. (2007). Suco de uva. Brasília: Embrapa Informação Tecnológica.

Robaskewicz, F., Dambrós, B. P., \& Santin, N. C. (2016). Determinação do teor de polifenóis totais e outras características físico-químicas em sucos de uva comerciais. Unoesc \& Ciência, 7(2), 159-166.

Sachi, A. T., \& Biasi, L. A. (2008). Maturação dos frutos de quatro cultivares de uvas muscadíneas em Pinhais, PR. Scientia Agraria, 9(2), 255-260. http://dx.doi.org/10.5380/rsa.v9i2.11015

Santana, M. T. A., Siqueira, H. H., Reis, K. C. D., Lima, L. C. D. O., \& Silva, R. J. L. (2008). Caracterização de diferentes marcas de sucos de uva comercializados em duas regiões do Brasil. Ciência e Agrotecnologia, 32(3), 882-886. http://dx.doi.org/10.1590/S1413-70542008000300027

Silva, G. G., Nascimento, R. L., Oliveira, V. S., Araújo, A. J. B., Oliveira, J. B., \& Pereira, G. E. (2011). Características físicoquímicas de sucos de uvas 'Isabel Precoce' e 'BRS Violeta' elaborados no Nordeste do Brasil. In Anais da $6^{a}$ Jornada de Iniciação Científica da Embrapa Semiárido (pp. 353-359). Petrolina: Embrapa Semiárido.

Sousa, J. S. I. (1996). Uvas para o Brasil. Piracicaba: Fundação de Estudos Agrários Luiz de Queiroz.

Threlfall, R. T., Morris, J. R., Howard, L. R., Brownmiller, C. R., \& Walker, T. L. (2005). Pressing effects on yield, quality, and nutraceutical content of juice, seeds, and skins from Black Beauty and Sunbelt grapes. Journal of Food Science, 70(3), 167171. http://dx.doi.org/10.1111/j.1365-2621.2005.tb07152.x

Threlfall, R. Y., Morris, J. R., Meullenet, J. F., \& Striegler, R. K. (2007). Sensory characteristics, composition, and nutraceutical content of juice from Vitis rotundifolia (Muscadine) cultivars. American Journal of Enology and Viticulture, 58(2), 268-273.

Toaldo, I. M., Cruz, F. A., Alves, T. L., Gois, J. S., Borges, D. L. G., Cunha, H. P., da Silva, E. L., \& Bordignon-Luiz, M. T. (2015). Bioactive potential of Vitis labrusca L. grape juices from the Southern Region of Brazil: Phenolic and elemental composition and effect on lipid peroxidation in healthy subjects. Food Chemistry, 173, 527-535. PMid:25466055. http://dx.doi.org/10.1016/j.foodchem.2014.09.171

Wei, Z., Luo, J., Huang, Y., Guo, W., Zhang, Y., Guan, H., Xu, C., \& Lu, J. (2017). Profile of polyphenol compounds of five muscadine grapes cultivated in the United States and in newly adapted locations in China. International Journal of Molecular Sciences, 18(3), 18. PMid:28335440. http://dx.doi.org/10.3390/ijms18030631 